Available online on 15.08 .2020 at http://jddtonline.info
Open Access to Pharmaceutical and Medical Research

Open $\odot$ Access

Research Article

\title{
Early Phasic Containment of COVID-19 in Substantially Affected States of India
}

\author{
Manisha Mandal', Shyamapada Mandal2, * \\ 1 Department of Physiology, MGM Medical College, Kishanganj-855107, India \\ ${ }^{2}$ Laboratory of Microbiology and Experimental Medicine, Department of Zoology, University of Gour Banga, Malda-732103, India
}

\begin{abstract}
Introduction: India is experiencing the global COVID-19 pandemic caused with the infection of SARS-CoV-2 (severe acute respiratory syndrome coronavirus 2). To explore the early epidemic course and the effectiveness of lockdowns on COVID-19 pandemic in some worstaffected Indian states.

Methods: Using publicly available real data and model-based prediction, the growth rate, case fatality rate, serial interval, and time-varying reproduction number (R) of COVID-19 were estimated, before and after lockdown implementation in India.

Results: The spread of COVID-19 epidemic in some highly-impacted Indian states displayed a characteristic sub-exponential growth projected up to 3 May 2020, as a consequence of lockdown strategies, in addition to improvement of reproduction number (R), serial interval, and daily growth rate, but not case fatality rate (CFR). The effect of COVID-19 containment was more prominent in second phase of lockdown with declining $\mathrm{R}$, which was still $>1$.
\end{abstract}

Conclusion: The current findings suggest the requirement of sustained interventions for effective containment of COVID-19 pandemic in Indian context.

Keywords: COVID-19, SARS-CoV-2, Indian states, epidemiological parameters, lockdown effect.

Article Info: Received 24 June 2020; Review Completed 14 July 2020; $\quad$ Accepted 20 July 2020; Available online 15 August 2020

\section{Cite this article as:}

Mandal M, Mandal S, Early Phasic Containment of COVID-19 in Substantially Affected States of India, Journal of Drug Delivery and Therapeutics. 2020; 10(4-s):31-33 http://dx.doi.org/10.22270/jddt.v10i4-s.4284

\section{*Address for Correspondence:}

Prof. Shyamapada Mandal, Laboratory of Microbiology and Experimental Medicine, Department of Zoology, University of Gour Banga, Malda-732103, India. Email: samtropmed@gmail.com

\section{INTRODUCTION}

Since the first detection of COVID-19 case on 30 January 2020, India reported 26,917 confirmed cases and 826 deaths; Maharashtra (7628) remains the worst affected state, followed by Gujarat (3071), Delhi (2625), and Rajasthan (2083), as of 26 April 2020 (MHFW-GoI, 2020). India, in an attempt to manage this crisis, enacted a twophase nationwide lockdowns, from 25 March 2020 to 14 April 2020, and thereafter extended up to 3 May 2020, because of the escalating spread of COVID-19 in India, in 32 states/union territories (Editorial, 2020; WHO, 2020). In view of this, we represent the early epidemic course of the disease in some highly-impacted Indian states, and its key features, using real data and model-based prediction, and explore the effectiveness of lockdowns in India on COVID-19 pandemic, since no such studies are available in India.

\section{METHODS}

Data on COVID-19, in Indian states, including Maharashtra, Delhi, Gujarat, Madhya Pradesh, Tamil Nadu, Rajasthan, and Uttar Pradesh (with $>1000$ confirmed cases, as of April 20, 2020), were retrieved electronically from publicly available domain of the Ministry of Health and Family Welfare, Government of India (MHFW-GoI, 2020), from 30 January 2020 through 20 April 2020 (MHFW-GoI, 2020); Kerala and West Bengal having case numbers $<1000$ were also included in the study. Daily growth rate (DGR), case fatality rate (CFR), serial interval (SI), and time-varying reproduction number (R) of COVID-19 cases were estimated, before and after lockdown, following the criteria mentioned earlier (Mandal and Mandal, 2020a). 


\section{RESULTS}

The spread of COVID-19 displayed a characteristic subexponential linear growth (mean DGR: 0.06; range: 0.01, for Kerala, to 0.11, for Gujarat, 95\% CI: 0.04-0.08, 11 April 2020 to 3 May 2020) that deviates from exponential growth estimates (mean DGR: 0.1643; range: 0.1163 , for Kerala, to 0.2175, for Tamil Nadu, 95\% CI: 0.1392-0.1894, 2 March 2020 to 3 May 2020), as a consequence of lockdown strategies (Figure 1). Reduction of $\mathrm{R}$, from a range of 1.352.86 (pre-lockdown 95\% CI: 1.49-2.21) to 1.13 - 1.67, in first phase, and to 1.08-1.63, in second phase of lockdowns (postlockdown 95\% CI: $1.20-1.42$ ), and cases by $56 \%-98 \%$, in individual states, have been reflected in our model estimates (Figure 1); $\mathrm{R}_{0}$ (basic reproduction number) among studied states ranged 1.24-1.96 (95\% CI: 1.36-1.78).

In addition, effectiveness of lockdown was revealed with improvement of SI (pre-lockdown: mean 3.82 days, 95\% CI: 2.55-5.1; post-lockdown: mean 14.26 days, 95\% CI: 2.44 26.08), DGR (pre-lockdown: mean 0.22, 95\% CI: 0.11-0.34; post-lockdown: mean $0.13,95 \%$ CI: $0.10-0.16)$, whereas CFR increased slightly (pre-lockdown: mean $2.54,95 \%$ CI: 0-5.52; post-lockdown: mean 3.11, 95\% CI: 1.19-5.03), as depicted in Figure 2.

\section{DISCUSSION}

In order to monitor and foresee the plausible progress of the ongoing COVID-19 pandemic, estimation of SI, CFR and $\mathrm{R}_{0}$ is of prime importance (Kupferschmidt, 2020), and daily deaths, and growth rate of COVID-19 cases also display importance in gaging the scale of the pandemic across time and space, and in framing policies and procedures (Leon et al., 2020; Yuan et al., 2020).
The dynamics of COVID-19 epidemic in Indian states with interventions was characterized by sub-exponential growth, which around 11 April 2020, deviated from the exponential growth of potential case numbers without interventions. Despite heterogeneities in socio-economic status and healthcare systems across Indian population (Editorial, 2020), similar growth patterns of COVID-19 among the states considered in the current study imply the effectiveness of the outbreak interventions.

Emergence of pre-symptomatic transmission likely occurred before lockdown in the states except Kerala, Uttar Pradesh, and Maharashtra, as explained by SI values lower than or almost equal to the incubation period ( $\sim 5$ days), while SI values higher than that of the incubation period indicated symptomatic transmission post-lockdown. Delhi and Uttar Pradesh displayed reduced post-lockdown DGR, while CFR increased in the states except Delhi and West Bengal. Maharashtra, Gujarat, and West Bengal curbed the disease spread more effectively compared to the other states with inflected post-lockdown $\mathrm{R}$ values, while West Bengal, Madhya Pradesh, Kerala, and Tamil Nadu projected greatly reduced case estimates (92\%-98\%) with lockdown, as on 3 May 2020, indicating better containment. The effect of COVID-19 containment was more prominent in the second phase of lockdown with declining $R$ values, which was still $>1$, suggesting further requirement of application of nonpharmaceutical interventions, in order to limit the SARSCoV-2 transmission (Pan et al., 2020; Kim et al., 2020), since no specific drugs are available for the treatment of COVID-19 (Mandal and Mandal, 2020b).

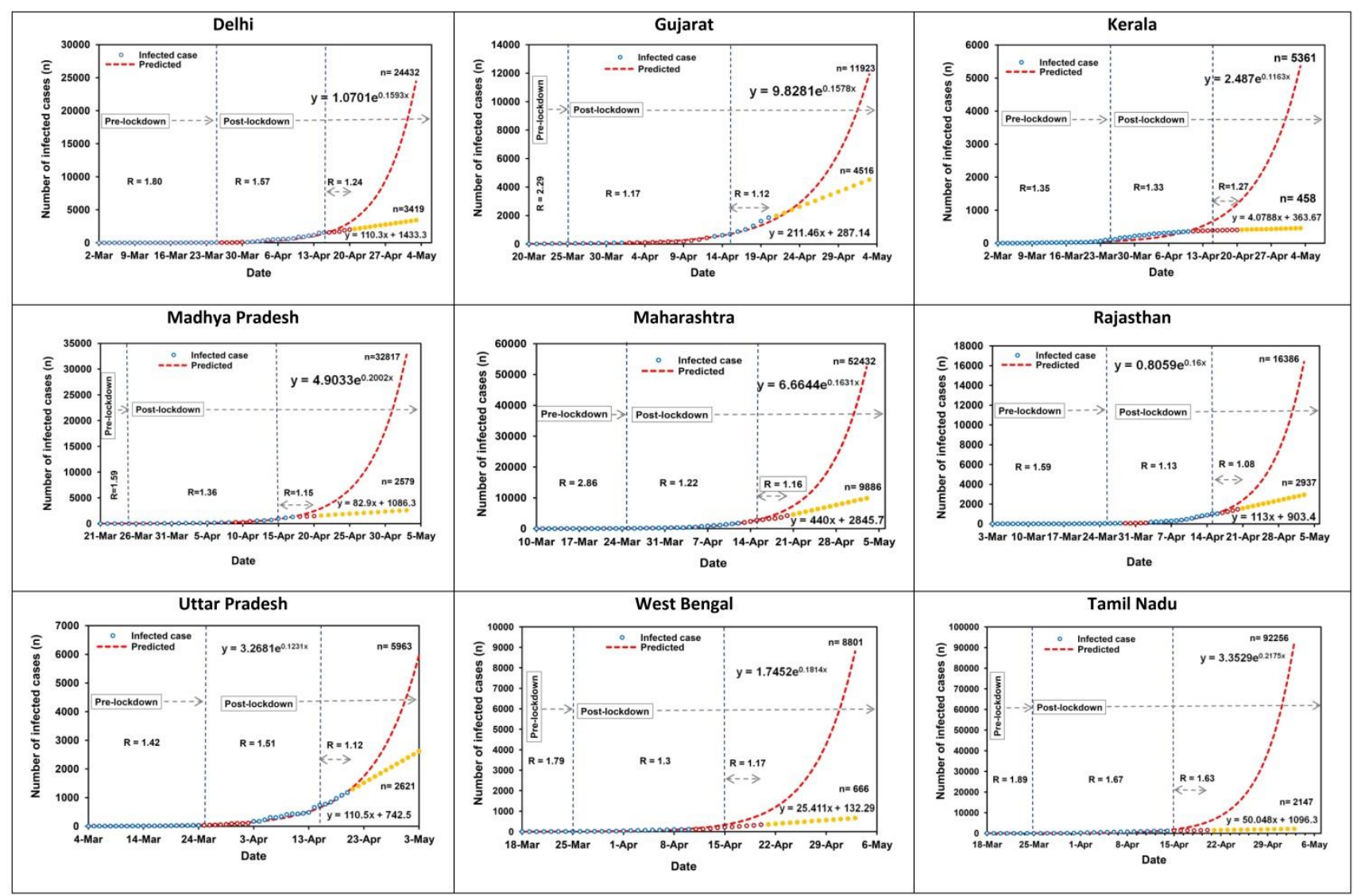

Figure 1: Data-based (as on April 20, 2020) and model estimation (up to May 3, 2020) of infected cases in Indian states. Red dashed lines denote estimated case numbers without lockdown, using exponential model. Circles represent observed infected case numbers with lockdown, forecast beyond April 20, 2020 (yellow solid circles) using linear model. Red hollow circles indicate lockdown-effective days, in two phases commencing on March 25, 2020 and April 15, 2020 (demarcated by dashed vertical lines). R: reproduction number. 


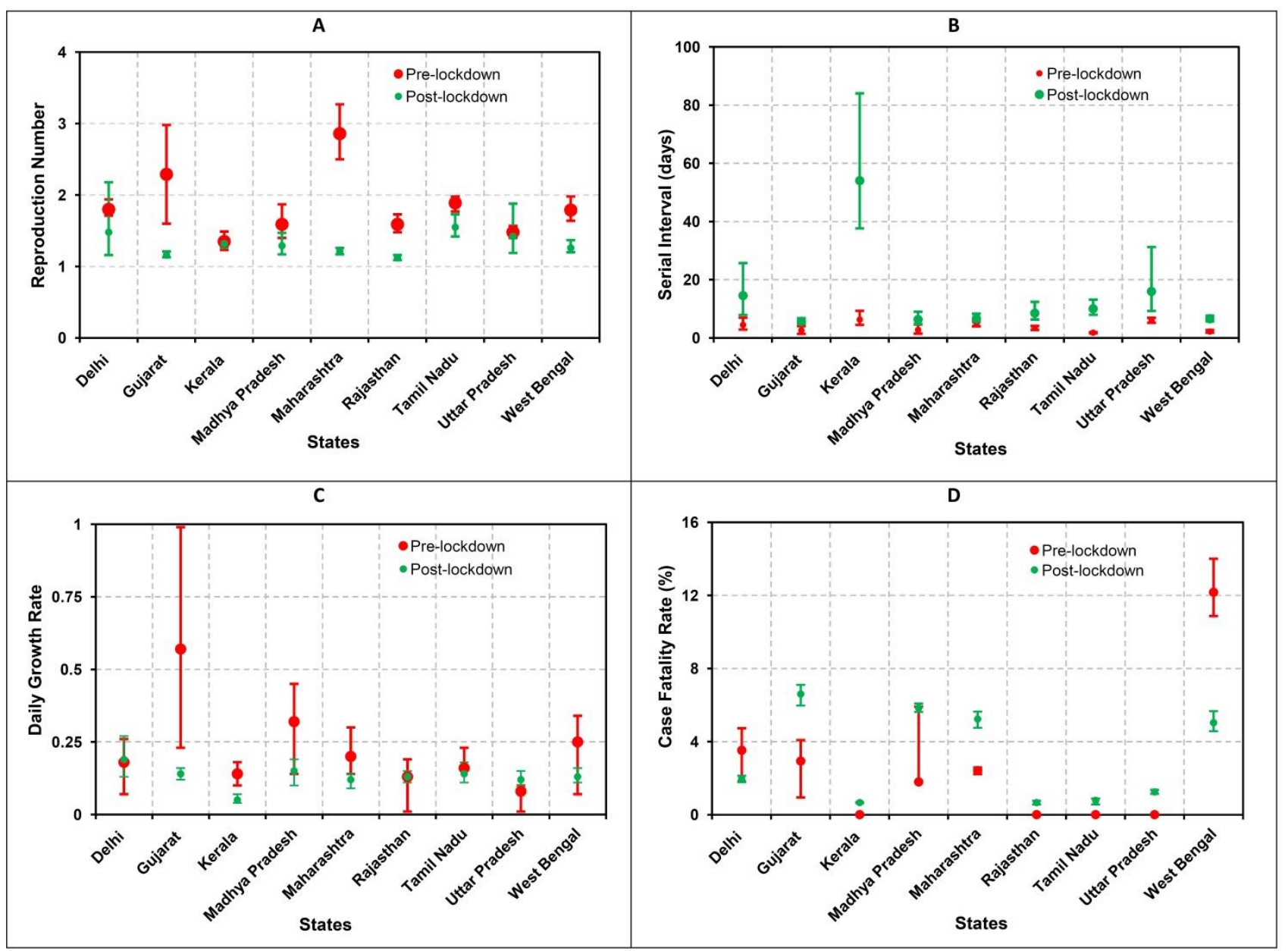

Figure 2: Epidemiological parameters of COVID-19 in Indian states before and after lockdown. A: reproduction number; B: serial interval; C: daily growth rate; D: case fatality rate. Vertical bars indicate standard errors of the mean.

\section{CONCLUSION}

The sustained lockdown measures, combined with testingisolating-contact tracing, face mask using and hygienepractice are need of the time for an early end of the ongoing COVID-19 pandemic in Indian context. However, since the pandemic develops real-time data availability will bring a clear reflection afresh.

Funding: There was no source of funding for this study.

Transparency declaration: [This article has been posted in medRxiv preprint, and] There are no potential conflicts of interest for any authors.

Authors' contribution: MM, Retrieved, processed and analyzed data, and wrote the paper; SM, designed the study, retrieved data, discussed and wrote the paper.

\section{REFERENCES}

1. Editorial. India under COVID-19 lockdown. Lancet 2020; 395:1315.

2. Kim GU, Kim MJ, Ra SH, Lee J, Bae S, Jung J, et al. Clinical characteristics of asymptomatic and symptomatic patients with mild COVID-19. Clinical Microbiology and Infection 2020; 26:948.e1-948.e3.
3. Kupferschmidt K. The lockdowns worked-but what comes next? Science 2020; 368:218-219.

4. Leon DA, Shkolnikov VM, Smeeth L, Magnus P, Pechholdova M, Jarvis CI. COVID-19: a need for real-time monitoring of weekly excess deaths. Lancet 2020; 395:e81.

5. Mandal M, Mandal S. COVID-19 pandemic scenario in India compared to China and rest of the world: a data driven and model analysis. medRxiv 2020a.

doi: 10.1101/2020.04.20.20072744

6. Mandal M, Mandal S. Bioinformatic approaches towards identification of potential repurposable drugs for COVID-19. Research Square 2020b. doi: 10.21203/rs.3.rs-38164/v2

7. MHFW-GoI. COVID-19 India. Ministry of Health and Family Welfare, Government of India. 2020. Available from: https://www.mohfw.gov.in/index.php (Accessed: 26 April 2020).

8. Pan A, Liu L, Wang C, et al. Association of public health interventions with the epidemiology of the COVID-19 outbreak in Wuhan, China. JAMA 2020; 323:1915-1923.

9. WHO India. Coronavirus disease (COVID-19). Available at: https://www.who.int/india/emergencies/novel-coronavirus2019 (Accessed: 20 April 2020).

10. Yuan Z, Xiao Y, Dai Z, Huang J, Chen Y. A simple model to assess Wuhan lock-down effect and region efforts during COVID-19 epidemic in China mainland. Bull World Health Organ. E-pub: 02 March 2020. doi: http://dx.doi.org/10.2471/BLT.20.254045 